Archivum, LXIV, 2014, pp. 239-274

\title{
Usos y valores modales del condicional en español
}

Recibido: 22/05/2014

Aceptado: 25/09/2014

\section{RESUMEN:}

En este trabajo, se analizan los distintos valores modales del condicional en español: el no factual, el de cortesía / atenuación, el de conjetura y el del rumor ("periodístico"). Tras volver sobre la historia de este tiempo, y sobre su posible naturaleza temporal o modal, se analizan los cuatro valores modales del condicional, mostrando que forman dos grupos: en el primero, que abarca el no factual y el de cortesía / atenuación, el condicional permite trasladar la acción a un mundo posible y conformar una construcción condicional del tipo <si p, q>; en el segundo, que abarca el de conjetura y el del rumor, el condicional permite trasladar la acción a un mundo posible y funciona como un operador epistémico de posibilidad: en el de conjetura, opera sobre el enunciado y en el del rumor, opera sobre la enunciación.

PALABRAS CLAVE: Condicional, modal, epistémico.

\section{ABSTRACT:}

This paper aims at explaining the different modal uses of conditional in Spanish: the hypothetical conditional, the mitigation / politeness conditional, the probability conditional and the journalistic conditional. In order to do this, I shall remember the history of this form, and then, how it has been considered as a tense or as a mood in Spanish grammars. My hypothesis is that two kinds of modal conditional in Spanish can be distinguished: for the first one, -ría refers to an action which is located in a possible world, and it is used in a conditional construction $\langle i f p, q\rangle$, with an explicit antecedent or an implicit one; for the second one, -ría also refers to an action located in a possible world but, this form can be considered as an epistemic operator of possibility: with the 
probability conditional, the possibility operates on a utterance and with the journalistic conditional, the possibility operates on the enunciation ( "illocutionary force").

KEY WORDS: Conditional, modal, epistemic.

\section{Introducción ${ }^{1}$}

El español dispone de cuatro usos modales de condicional llamados "usos modalizados de los tiempos" (NGLE2 2009: §25.1f), por lo general compartidos por los estudiosos (NGLE 2009: §25.1f, Gili Gaya 1993: §129, Rojo \& Veiga 1999: §44.3.3, entre muchos otros). En términos dela NGLE (2009: §25.1f), estos usos modales son: el condicional no factual, el condicional de cortesía o de atenuación, el condicional de conjetura y el condicional del rumor. A diferencia del español, otras lenguas románicas carecen de uno de ellos. A modo de ilustración, se advertirá que el condicional de conjetura no existe ni en francés ni en italiano. Ahora bien, el ser "modal" ha recibido un número amplísimo de definiciones de forma que tan solo retendremos dos. Desde el punto de vista de la gramática, según la NGLE (ibíd.), el significado de cada uno de estos condicionales modales "se obtiene subordinando implícitamente sentidos temporales a algún predicado de naturaleza epistémica que denota probabilidad, suposición u otras nociones semejantes que relativizan (o convierten en dependiente) el estado de cosas que se considera". Rojo \& Veiga (1999: 2915) por su parte, aluden a la noción de "improbabilidad" (de realización improbable) o de "irrealidad". Ser modal, por tanto, significa subordinar el sentido temporal a algún tipo de predicado que expresa la probabilidad / improbabilidad u otra noción que permita relativizarlo.

1 Este trabajo fue presentado el 12 de mayo de 2014 en el departamento de Lengua Española, Teoría de la Literatura y Literatura comparada de la Universidad Complutense de Madrid, bajo la forma de una conferencia. Quiero expresar mis más sinceros agradecimientos a Ignacio Bosque, Cristina Sánchez, Luis García, Manuel Leonetti y José Luis Girón Alconchel, quienes, por sus valiosos comentarios, me ayudaron a mejorarlo. Agradezco también los acertados comentarios que me han hechos dos revisores anónimos.

2 Nueva Gramática de la Lengua Española. 
En términos de la lógica modal,la modalidad, al tratar de hechos que no ocurren en el mundo actual ya que son de naturaleza posible o probable, se define a partir de los mundos posibles, esto es, a partir de otro mundo distinto del mundo actual (Kratzer 1991). Una oración, o un verbo, adquiere valor modal cuando describe una situación que se da en otro mundo - un mundo posibleque no es el actual. Así como dice Kearns (2011: 8), “[t]he term possible worlds is used in semantics for hypothetical ways reality might be or might have been. (...) A possible world different from the actual world is a whole alternative universe". Las dos modalidades que se manejan con mayor frecuencia ${ }^{3}$ son la modalidad epistémica y la modalidad radical/deóntica. Una definición muy clásica es la de Lyons (1977: 793 \& 823): la modalidad epistémica es proposicional, ya que tiene que ver con el conocimiento del hablante sobre la posibilidad o la necesidad de la verdad de una proposición, mientras que la modalidad deóntica se relaciona con la posibilidad o la necesidad de los actos realizados por un indivi$\mathrm{duo}^{4}$. Numerosos autores recurren a la noción de probabilidad o de modalidad epistémica para caracterizar al condicional modal (NGLE 2009: §23.15j; Rojo \& Veiga 1999, entre otros).

Nuestro objetivo será exponer de qué forma se manifiesta la modalidad en los condicionales modales: el no factual, el de probabilidad, el del rumor, el de cortesía, destacando los rasgos que permiten orientar la lectura de un enunciado hacia un determinado tipo de condicional modal. Unas posibles preguntas son: ¿se manifiesta lo modal de la misma forma en los cuatro tipos de condicional? ¿Aparecen la probabilidad / la irrealidad, o se relativiza el estado de cosas de un mismo modo en los cuatro tipos de condicional? En segundo lugar, ¿es razonable esa divi-

3 Decidimos simplificarlo por no entrar en la cuestión, muy debatida, del número de modalidades que pueden aparecer en la lengua, y en segundo lugar, porque en la lógica modal, en principio, sólo existen esas dos: epistémica y radical/ deóntica (véase Laca 2005: 44).

4 Véase también NGLE (2009:§ 28.6c). 
sión cuadripartita o bien estos cuatro usos del condicional tienen puntos en común que permiten agruparlos en dos únicas categorías? En nuestra opinión, las nociones de "probabilidad", de "dependencia" o de "modalidad epistémica" a las que se suele recurrir en la bibliografía no dan cuenta de las diferencias que existen entre los cuatro tipos de condicionales modales.

Nuestra hipótesis es la siguiente: se puede agrupar los cuatro condicionales en dos clases. Intentaremos mostrar que la modalidad se manifiesta de dos formas distintas en estos condicionales en español. Siguiendo la idea según la cual un tiempo puede definirse como un operador que tiene bajo su ámbito una proposición entera (Kearns: 2011: 185; Laca 2005; Escandell 2004: 244; 251), defenderemos la idea de que la flexión de condicional -ría funciona como un operador modal hipotético de dos formas: en el caso del condicional no factual y del de cortesía /atenuación, el morfema -ría sirve para trasladar la situación a un mundo posible y conformar una construcción condicional del tipo $<$ si $p, q>$. En el caso del condicional de conjetura y del de rumor, el morfema -ría hace de operador epistémico de hipótesis, esto es, traslada la situación a un mundo posible, pero sin conformar ninguna condición. Más bien, mediante este morfema, el hablante expresa su punto de vista hipotético sobre el enunciado (lo dicho) o sobre la enunciación (compromiso del hablante con lo dicho).

Para analizarlo, en la primera parte, daremos un repaso a la historia de este tiempo cuyo origen radica en una perífrasis de contenido modal; en la segunda parte, recordaremos, cómo a lo largo de la historia, se ha considerado unas veces modo y otras veces tiempo; en la tercera parte, volveremos sobre el valor temporal del condicional; en la cuarta parte, se analizarán el valor modal del condicional no factual y el del condicional de cortesía; por fin, en la última, estudiaremos el valor modal del condicional de conjetura y el del condicional de rumor. 


\section{Repaso histórico: del latín al castellano medieval}

Como bien se sabe, el condicional es una creación románica que vino a sustituir al imperfecto y al pluscuamperfecto de subjuntivo latino. El origen románico de este tiempo es una perífrasis creada a partir del infinitivo del verbo (CANTARE) y del imperfecto de HABERE (HABEBAM) que se convirtió en una forma sintética, tal y como cantaría en español, canterei en italiano, cantaría en portugués, chanterais en francés. El español lo conforma de la siguiente forma: de una forma perifrástica o analítica, pasamos a una forma sintética: CANTARE + habebam > cantar(h)íal-ie.

El futuro ya existía en latín mientras que el condicional es de creación nueva. Sin embargo, en las gramáticas históricas, se suelen estudiar conjuntamente (Company Company 2006, Penny 2001, Lloyd 1993). Difícilmente se puede fechar su aparición oral: según Company Company (2006: 349), aparece la nueva forma de futuro en el s. I a.C. Otros como Benveniste dicen que aparece en el s. III. La primera forma sintética de futuro, como creación románica, aparece por escrito en el s. VII en Crónica de Fredegario (Crónica desde la creación del mundo hasta Carlomagno):Et ille respondebat: "Non dabo". Iustinianus dicebat: "Daras". En cuanto al condicional español, suele decirse que aparece más tarde, en el s. XI, en las Glosas Emilianenses. Cabe notar una diferencia en la formación del futuro puesto que, si bien el italiano,(canterò), el portugués (cantarei), el francés (chanterai) se forman a partir del presente del auxiliar HABERE, el rumano se forma a partir del presente de VOLERE y conserva su forma perifrástica hasta hoy en día (voi cânta). Ambas perífrasis, tanto la de futuro como la de condicional, tenían un sentido de obligación (Company 1985; Girón Alconchel 1997). Por tanto, la nueva forma de futuro significaba algo próximo a he de cantar y la de condicional, del mismo modo, a había de cantar.

Una de las preguntas recurrentes de los historiadores es saber por qué tanto para el futuro como para el condicional se recurrió a una forma perifrástica con sentido de obligación. Los argumen- 
tos comunes para ambas formas son los siguientes: Ya existían numerosas perífrasis en latín para sustituir al futuro sintético latino AMABO: facere incipio, facere uolo, facere debeo, así como formas participiales facturus sum y pasivas faciendus est. Por otra parte, el futuro parecía bastante inestable, al conformarse de dos formas distintas, en -bo (CANTABO) y en -am/-em (LEGAM) de modo que se quería evitar posibles confusiones entre la segunda forma de futuro y el subjuntivo, entre el pretérito indefinido CANTAVIT y el futuro CANTABIT, los cuales llegaron a pronunciarse de forma igual. Otras explicaciones son la tendencia al abandono de las formas sintéticas en provecho de las formas analíticas en el paso del latín al español, y la nueva forma de ver el porvenir ligado a la Cristiandad así como dice Company Company (2006: 354):

La futuridad ya no es entendida como un simple hecho temporal existente con independencia de las acciones del ser humano, sino que es una futuridad modal obligativa, en el sentido de que el individuo es responsable de su propio futuro y puede modificarlo a través de sus acciones en el presente.

En todas las lenguas románicas, por tanto, se da el mismo fenómeno.Veremos ahora cómo funcionaba en latín y a continuación, daremos un breve repaso al castellano medieval. En latín, coexistieron el futuro analítico (CANTARE + HABERE) con el futuro sintético (CANTABO) durante más de 4 siglos, del s. III al s. VII d.C, de modo que se ha llegado a destacar una diferencia de sentido entre ambas formas; el futuro sintético expresaba más bien la obligación personal o individual, mientras que el futuro analítico o perifrástico expresaba tan solo la obligación impersonal o proposicional. CANTABO contenía un componente de intencionalidad y por tanto se podía traducir por "yo tengo intención de cantar", mientras que CANTARE HABEO carecía de la noción de intención y tan solo expresaba la necesidad epistémica, esto es, "es necesario que yo cante". Ocurría lo mismo con el condicional, el cual, al ser una forma perifrástica, expresaba una necesidad epistémica de pasado: "era necesario que esto ocurriera". Esto explica 
el que el condicional en latín se encuentre a menudo en construcciones pasivas: "[...] a quibus [...] suscipi habebat" (Tertuliano, s. III) (Merle 2001: 24), no tenía el sentido de "por los cuales tenía intención de ser recibido" sino más bien "por los cuales era necesario que lo recibieran", entendiendo que su destino era que lo recibieran. Por tanto, en latín, el condicional se ha formado a partir de una perífrasis de significado modal, que, por operar sobre una proposición, expresaba la noción de necesidad epistémica.

En castellano medieval, se ha observado que las dos formas analíticas (perifrásticas) y sintéticas coexistieron durante mucho tiempo ya que las formas analíticas se documentan hasta el s. XVII. Pasa lo mismo con el catalán y el portugués pero no ocurre con el francés (Castillo Lluch 2002: 541).Los futuros analíticos (perifrásticos) aparecen por razones semánticas, esto es, cuando claramente predomina el valor modal. De ese modo Company Company (2006: 356) dice que "con los condicionales analíticos, el valor modal de probabilidad y deseo predomina sobre la temporalidad". Por otra parte, puede usarse por razones estrictamente sintácticas ya que "estaba prohibido que un pronombre átono apareciera en posición inicial de la oración" de modo que podíamos encontrar ejemplos del tipo:

(1) Senora, quién vos diese una cibdad tamana como Roma quando estva en su éser,..., amarlo yades senora?(Corbacho 122, in Company Company 2006: 409).

Para explicar la coexistencia de las dos formas, los historiadores han considerado que el condicional en español no se había gramaticalizado del todo (Menéndez Pidal 1926; Penny 2001) o bien, han desarrollado la idea de que el uso de las formas analíticas iba asociado con un efecto de énfasis discursivo (Company y Medina 1999).

Las formas sintéticas del condicional, en cambio, alternan con el imperfecto de subjuntivo, lo que, una vez más, resalta su alto carácter modal (Pato 2003; Company Company 2006), como se podrá comprobar a continuación: 
(2) E estaua el conde jocelin con su muger \& fijos dentro en el castillo. E el conde quando se vio cercado ouo gran miedo que tomarian el castillo \& que prenderian a el \& a la muger \& a sus fijos \& quantos dentro estauan [Granconquista de Ultramar].

(3) mando combatir el castillo de todas partes \& penso le tomar a poco tiempo: ca no se temia que le leuantassen de la cerca: porque los mayores hombres del reyno estauan dentro con el rey [Gran conquista de Ultramar].

En este primer capítulo, hemos intentado volver sobre el origen de la forma de condicional. La perífrasis que lo conforma significa obligación, esto es, necesidad epistémica, y por tanto, se observa que desde el principio, cobra un valor modal. Esto hace que a lo largo de la historia, haya habido un largo debate entre los gramáticos sobre la naturaleza temporal o modal del condicional. Vamos a verlo a continuación.

\section{2. ¿Modo o Tiempo?}

La idea de que el condicional es modo radica en un criterio semántico, mientras que la idea de que el condicional es tiempo radica en un criterio sintáctico.

\subsection{Modo: criterio semántico}

Dos ideas básicamente subyacen a la idea de que el condicional es modo. La primera es aquella según la cual cualquier enunciado expresado en condicional está subordinado explícitamente o implícitamente a una subordinada de condición. La segunda es aquella según la cual el condicional permite considerar la acción como hipotética sin situarla en un eje cronológico, con lo cual se asemeja a los modos más que a los tiempos. Para ilustrarlo, se observa que Elio Nebrija, el primero en hablar del condicional, en su Gramática Castellana de 1492, adopta la clasificación siguiente: tiempos verbales en el modo indicativo: presente, imperfecto; tiempos 
verbales en el modo optativo: subjuntivo, imperfecto; tiempos verbales en el modo subjuntivo: condicional, y el imperativo. En el siglo XX, la gramática de la RAE de 1917 ha creado un modo -modo potencial- para el solo condicional (1917: §285). Más adelante, la gramática de la RAE (1973: §3.14.9c) admite que es tiempo pero subraya su carácter altamente hipotético diciendo que:

Por su carácter de tiempo futuro, la acción que expresa [el condicional] es siempre eventual o hipotética, como en todos los futuros.

\subsection{Tiempo: criterio sintáctico}

Andrés BELLO (1847: § 454) considera el 'pos-pretérito' como un tiempo: "Siendo el régimen lo que verdaderamente distingue los Modos, sólo por él podemos clasificarlos y definirlos". Muestra que surge en los contextos en los que se usan tiempos del indicativo:

(4) \{Sabía/afirmó\} que tus intereses \{prosperarían/prosperaban/prosperaron $/{ }^{*}$ prosperen $/{ }^{*}$ prosperaran $/{ }^{*}$-sen\}.

Y no como los tiempos del subjuntivo:

(5) Dudaba que $\left\{{ }^{*}\right.$ te gustaría / *te gustaba / *te gustó/ guste/ gustara / -se\}.

La NGLE (2009: §23.15) retoma los argumentos de Bello (1847) explicando que "el condicional aparece en entornos sintácticos en los que se selecciona el modo indicativo". Hoy por tanto, suele aceptarse la idea de que es el condicional un tiempo con valor temporal y con valores modales. Recordemos brevemente lo que puede entenderse por condicional temporal.

3. Valor temporal: "condicional factual o narrativo" (NGLE 2009: 23.15r)

Un condicional temporal es un futuro en el pasado porque designa un hecho posterior a otro hecho pasado. Es un tiempo 
relativo porque sitúa la acción respecto del momento del habla y respecto de otro punto del pasado.

(6) Ayer, Juan dijo que llegaría hoy a las 2:00.

En el ejemplo (6), llegaría se sitúa después del acontecimiento pasado dijo. En cambio, no se le puede situar respecto del origen con exactitud: puede haber llegado antes del presente de la enunciación, en el presente o después. Si bien la relación con el momento del pasado es fija, "la relación con el momento de habla es indeterminada" (Molina Redondo 2011: 171). Con esto, se observa que resulta ser un tiempo sumamente ambiguo porque designa una acción de naturaleza futura, la cual, por tanto, en principio, no ha ocurrido, y a la vez una acción que, por ser o no pasada, puede o no haberse realizado. Al ser un futuro-respecto de un punto del pasado y no respecto del presente del habla-, no puede tener uno la certeza-como ocurre con el futuro-de que no haya ocurrido la acción.

La segunda cuestión es, ¿cómo identificarlo? Si se puede sustituir la forma de condicional por la perífrasis 'ir + infinitivo' en pretérito imperfecto, es un condicional con valor temporal ${ }^{5}$. Observamos que esta sustitución es posible en (7) pero no en (8) con un condicional de conjetura ni en (9) con un condicional de atenuación.

(7) a. Ayer, Juan dijo que llegaría hoy a las 2:00.

b. = Ayer, Juan dijo que iba a llegar hoy a las 2:00

(8) a. En aquella época, Juan tendría unos 40 años.

b. \# En aquella época, Juan iba a tener unos 40 años ${ }^{6}$.

(9) a. Deberías estudiar más.

5 Este criterio lo introduce Haillet (2002) para el francés y la NGLE (2009: §23.15j) para el español.

6 Se considerará que el símbolo \# significa 'no equivale a, no puede sustituirse por'. 
b. \# Ibas a deber estudiar más.

Abordaremos a continuación los diferentes valores modales del condicional en español que son, como ya se ha dicho: el no factual, el de cortesía / atenuación, el de conjetura y el del rumor. Empecemos por el condicional no factual.

\section{El condicional no factual y el condicional de cortesía / de atenuación.}

\subsection{El condicional no factual}

A este condicional se le llama condicional no factual porque se usa en contextos "no factuales", "es decir, no reales, no verificados o no experimentados" (NGLE 2009 § 25.1c). Para simplificarlo, puede afirmarse que sirve para aludir a los "no hechos", es decir a las cosas que no ocurren. Expresa "contenidos no factuales supeditados a situaciones hipotéticas" (NGLE 2009 \$23.15) y por tanto, sintácticamente, se construye con una subordinada (prótasis) explícita o implícita. Vamos a ver cada una de estas dos construcciones.

Con una subordinada explícita, abarca tanto a las construcciones condicionales potenciales (10) como a las condicionales irreales (11) y (12). En el primer caso, el hablante considera que la hipótesis tiene posibilidades de realizarse. En el segundo caso, el hablante considera que la hipótesis no las tiene?

(10) Si hablaras con él, se arreglarían las cosas.

(11) Si hubieras hablado con él, se habrían arreglado las cosas.

(12) Si los hombres fueran mujeres, todo sería fácil.

7 Es un hecho ya muy consabido que la diferencia entre las potenciales y las irreales no puede explicarse sino por factores pragmáticos (véase Ridruejo 1989: $\S 7.7)$. 
Las potenciales y las irreales tienen puntos comunes y también diferencias. Su punto en común es que siguen el mismo patrón, $<$ si $p, q>$ : la realización de $q$ (apódosis o consecuente) depende de la de $p$ (prótasis o antecedente). El valor de verdad de arreglarse las cosas, depende del de hablar tú con él. Es el valor de verdad ${ }^{8}$ de la prótasis el que determina el valor de verdad de la apódosis de modo que $<$ si $p$ es verdad, $q$ es verdad $>$ y $<$ si $p$ no es verdad, $q$ no es verdad $>$. Algunos dicen como W.V. Quine (1959:12) que el elemento realmente hipotético es el consecuente: "An affirmation of the form 'if $p$, then $q$ ' is commonly less as an affirmation of a conditional than a conditional affirmation of the consequent". Dicho de otro modo, $p$ y $q$ se sitúan en un mundo posible, que es otro mundo distinto del mundo actual, y por esta razón adquiere el enunciado un valor modal. Cabe distinguir entre mundo actual y mundo posible. Ni $p$ ni $q$ se aseveran en el mundo actual. Lo que se asevera en el mundo actual es la relación de condición introducida por si, esto es, la relación de necesario entrañamiento (implicación) entre $p$ y $q$. Vamos a ver ahora en qué difieren las potenciales y las irreales.

Retomemos el siguiente ejemplo:

(13) Si hablaras con él, se arreglarían las cosas.

Para que cobre valor de verdad $\langle$ si $p, q\rangle$, se observa que $p$ puede ser verdad o no, y que $q$ puede ser verdad o no, conque tendríamos:

(14) Si hablas con él, se arreglarán las cosas.

(15) Si no hablas con él, no se arreglarán las cosas.

Esto significa que no importa que hables o no con él ni que se arreglen o no las cosas para que la frase Si hablaras con él, se arreglarían las cosas tenga valor de verdad. Se sigue manteniendo la relación de entrañamiento entre $p$ y $q$. Esta interpretación tiene

8 Se recuerda que tal y como dice G. Frege (1971: 109-110), el valor de verdad de una proposición es lo verdadero o lo falso. 
que ver con el conocimiento del hablante, el cual en su presente considera que la acción es de realización posible. El conocimiento del hablante sobre el mundo posible y el que tiene sobre su presente son compatibles.

En el caso de las condicionales irreales, cabe distinguir entre las de pasado y las de presente, "past counterfactual" y "present counterfactual”, según Iatridou (2000: 231-232).

(16) Si hubieras hablado con él, se habrían arreglado las cosas.

En las irreales de pasado, como en (16), $p$ y $q$ deben ser falsas para que $<$ si $p, q>$ cobre valor de verdad. Para que la frase (16) tenga sentido (valor de verdad), es obligatorio que 'no hayas hablado con él' y que 'no se hayan arreglado las cosas'. Dicho de otro modo, el hablante en su presente considera que en el presente, no se da la situación, que en el futuro, la acción es de realización imposible y que en el pasado, no se ha realizado la acción ( $<$ no $p>y<$ no $q>)$. Desencadena una implicatura que es una presuposición de pasado 9 .

En las irreales de presente, como en (17), también $p$ y $q$ deben ser falsas para que $<$ si $p, q>$ cobre valor de verdad.

(17) Si los hombres fueran mujeres, todo sería fácil.

El hablante desde el presente de habla considera que en el presente no se da la situación y que en el futuro es de realización imposible. Desencadena también una presuposición de negación, pero solo de presente y no de pasado como en el caso anterior (implica los hombres no son mujeres y no *los hombres no han sido mujeres). En muchos casos, las irreales se construyen con verbos de estado que describen una propiedad inalienable del individuo ya que, como se recordará, desde el punto de vista aspectual, los estados, al no tener ni principio ni final, no pueden 
ocurrir ni realizarse ${ }^{10}$. En dicho caso, el conocimiento del hablante sobre el mundo posible no es compatible con su conocimiento del mundo presente.

Esta definición de las irreales tiene dos consecuencias. La primera es que son enunciados contrafácticos (Stalnaker 1968; Iatridou 1998, 2000, Moss 2012, von Fintel 2001), de los que se han dado las definiciones siguientes:"counterfactuals as literal statements about non actual situations" (Stalnaker 1968: 111), "contrary to fact" (Iatridou 2000: 231), "situaciones manifiestamente contrarias a los hechos tal y como son en el mundo real" (Escandell 2004: 297).

La segunda es que no son verdaderas condicionales o al menos, hace falta matizarlo. Como indica Stalnaker (2011: 234), Grice ya notó que era una equivocación hablar de condicionales basándose en la falsedad del antecedente ${ }^{11}$. Montolío (1999: 3647-3648) retoma la idea diciendo que aunque las irreales expresan una condición, no expresan una hipótesis. En efecto, una condición es un enunciado del tipo $<$ si $p, q>$ y para que surja la hipótesis, cabe la necesidad de que exista una alternativa: sí o no. Ahora bien, al presuponer las irreales la negación del hecho, desaparece la alternativa y se expresa una condición pero no una hipótesis estricto sensu. El que no haya ocurrido o no ocurra es una implicatura: solo puede definirse una construcción como hipotética a partir de factores pragmáticos Significa que gramaticalmente, todas, tanto las potenciales como las irreales son condicionales, pero pragmáticamente, algunas son hipótesis (potenciales) y otras no lo son (irreales).

Existen por otra parte condicionales que aparecen sin prótasis, para la comprensión de los cuales es necesario sobreentender una subordinada:

10 Aquí tenemos uno de los pocos criterios no pragmáticos sino aspectuales que permiten orientar la lectura de la construcción condicional.

11 "As Grice noted, it is normally misleading to assert a (material) conditional on the basis of knowledge of the falsity of the antecedent $(\ldots)^{\prime \prime}$ 
(18) Maite dejaría su trabajo a la primera oportunidad (si pudiese).

(19) Ahora cumpliría 60 años (si estuviera vivo).

(20) Juan dimitiría (si pudiese).

Lo que se observa es que comparten dos propiedades: la de obedecer a la construcción $\langle$ si $p, q\rangle-$ encontramos también potenciales e irreales- y en segundo lugar, la de usarse con la tercera persona. No parece ser una observación de mayor relevancia y sin embargo, vamos a ver que sí lo es. Si retomamos el ejemplo (20) Juan dimitiría, cambiamos la persona y decimos:

(21) Yo dimitiría.

A la primera se suma una segunda interpretación. En la primera, expreso mi deseo de dimitir, subordinado a circunstancias: "dimitiría si pudiese, si tuviese dinero" y por tanto, se lee como un condicional no factual. Sitúo la acción de dimitir en otro mundo, subordinada a una prótasis implícita. Existe una segunda interpretación. De este condicional no factual $\langle$ si $p, q\rangle$, se saca una implicatura de presente, esto es, un consejo o una petición"dimite" o "te aconsejo que dimitas"; le pido al interlocutor de un modo indirecto que actúe de una determinada forma y aparece el acto de habla indirecto: condicional de cortesía o de atenuación ${ }^{12}$. Es esta misma implicatura la que le da su sentido al enunciado. Se reinterpreta pragmáticamente. Para resumir, el condicional no factual es modal y obedece al patrón $\langle$ si $p, q\rangle$ (con una subordinada explícita o implícita), trasladando la acción a un mundo posible distinto del mundo actual. Vamos a ver ahora más detenidamente cómo se debe entender el condicional de cortesía o atenuación.

12 Véase Rivero (1978: 79), la cual alude a la fuerza ilocutiva de ruego que llega a tener el condicional de cortesía. Como se verá a continuación, la teoría de "los actos de habla" de Grice (1975) y de Searle (1969) es la que le da al condicional su valor de cortesía porque se interpreta como un "acto indirecto". En este caso, "la interpretación de un acto indirecto es resultado de una implicatura y se obtiene por inferencia" (Escandell 1995: 36). 


\subsection{El condicional de cortesía y de atenuación.}

El condicional de cortesía o de atenuación suele considerarse como un determinado tipo de condicional modal. Lleva distintos nombres: "condicional de cortesía y condicional de mitigación" (Gili Gaya 1993: 169); "de atenuación"(Haverkate 1994: 144-145; 185; 192); "de modestia o de cortesía" (NGLE 2009: §23.15ñ). Se le llama así porque permite atenuar la fuerza de una petición o de una afirmación. Esto explica que la noción de cortesía, aquí expresada mediante el condicional, se formule a partir de la "teoría de los actos de habla" (Searle 1969, Grice 1975): una frase puede interpretarse literalmente, o bien a partir de una inferencia que es el acto que uno realiza al pronunciar un enunciado (por ejemplo, un ruego, una petición). Seguiremos la idea defendida por Rivero (1978) y Veiga (1991) según la cual el condicional de cortesía o de atenuación es un condicional no factual (potencial o irreal) pero intentaremos mostrar que el significado de cortesía o de atenuación se obtiene mediante un acto verbal que es una implicatura de presente. Lo primero que hace falta recalcar es que se obtienen la cortesía y la atenuación a nivel de la enunciación ${ }^{13}$. Cabe distinguir entre cortesía y atenuación: la cortesía va enfocada hacia el interlocutor y permite "manifestar la consideración y respeto hacia los interlocutores" (Albelda \& Briz 210: 238) mientras que la atenuación va enfocada hacia el hablante y "consiste en minimizar la fuerza ilocutiva de los actos de habla" (Albelda \& Briz 210: 238). Los dos tipos de enunciados producen una inferencia que consiste en una petición de acción: en el primer caso, la petición de acción es "actúa de una determinada forma" y en el segundo caso, es "acepta lo dicho". Llamaremos, a los del primer tipo, condicionales de cortesía, y a los del segundo, condicionales de atenuación. Observemos los ejemplos (22) a (26).

13 Así como afirma Kerbrat-Orecchioni (2004: 47) “la cortesía no es una propiedad de las oraciones (en inglés sentences) sino de los enunciados (en inglés utterances): fuera de contexto, ninguna secuencia puede ser calificada "cortés" ni "descortés"." 
(22) ¿Me podrías acercar la fuente?

(23) Me encantaría que me acompañaras al cine.

(24) Me gustaría que me acompañaras al cine.

(25) De buena gana me tomaría un café.

(26) ¿De qué color es este jersey? -Yo diría que es azul14.

Vamos a intentar mostrar que (22) a (24) son condicionales de cortesía porque producen un acto de habla indirecto que es una petición de acción mientras que (26) es un condicional de atenuación cuyo único objetivo ilocutivo es que el interlocutor acepte lo dicho ${ }^{15}$. Cada uno de estos enunciados tiene una lectura literal y una lectura inferida o descodificada (implicatura) (Rivero 1978; Escandell 1995). (22) literalmente significa "eres capaz de acercarme la fuente" o "ite es posible acercarme la fuente?" y conlleva una implicatura, esto es, una petición indirecta: "acércame la fuente"16. La segunda lectura es la que le da su sentido a la frase ya que lo que espera el hablante no es una respuesta por "sí" o "no", sino una determinada acción. En (23) la lectura literal sería "si me acompañaras al cine, me encantaría" y conlleva una implicatura, una petición indirecta, "acompáñame al cine". (24) literalmente significa "si me acompañaras al cine, me gustaría" y desencadena la implicatura "acompáñame al cine". (25) se entiende literalmente como "si pudiese, me tomaría un café" y en función del contexto, se saca una posible implicatura que es "tráeme un café". Y por fin, (26) puede entenderse como"si me lo preguntaras, te diría que es azul" e implica"acepta lo que digo", de modo que aparece una petición de acción que consiste en pedir que se acepte lo dicho. Solo atenúa lo que se está diciendo. En este caso, la enunciación (decir) coincide con el enunciado (diría). Se observará

14 Este ejemplo está sacado de Carrasco Gutiérrez (1999: 3097).

15 Tal y como dice Briz (2004: 69) “la atenuación, [...], es una operación lingüística de minimización de lo dicho y del punto de vista, que afecta, así pues, al valor intencional, a la fuerza ilocutiva".

16 Véase para mayor detalle Escandell (1995: 34, 37 y 50). 
que este condicional de atenuación se conforma con verbos de lengua tal y como decir, añadir, rogar, pedir.

Al obtenerse el sentido de atenuación y de cortesía a nivel de la enunciación, se ha comentado que dicho condicional equivale a un presente (NGLE 2009: $§ 23.15$ n).Vamos a intentar comprobar si se confirma.

(27) a. De buena gana me tomaría un café.

b. \# Ahora mismo me estoy tomando café.

c. $=$ Ahora mismo deseo que me traigas uno.

(28) a. Deberías estudiar más \{ahora mismo / *ayer por la tarde\}.

b. = Ahora mismo debes estudiar más.

(29) a. Me encantaría que me acompañaras al cine \{ahora mismo / *ayer por la tarde\}.

b. \# Me encanta que me acompañes al cine.

(30) a. Me gustaría que me acompañaras al cine \{ahora mis$\mathrm{mo} /$ *ayer por la tarde\}.

b. \# Me gusta que me acompañes al cine.

En (27) no se da la equivalencia con el presente porque lo que es presente no es la misma acción de 'tomarse un café' sino 'el deseo de tomarlo'; en (28), sí el verbo modal permite la equivalencia con el presente; por fin, (29) y (30) no equivalen a un presente sino que además, en presente pueden también adquirir un sentido genérico que no tienen en el enunciado condicional. Para resumirlo, no siempre equivale a un presente porque la situación descrita por el verbo en condicional no se sitúa en el presente sino en otro mundo posible. Lo que sí es presente, es la implicatura, esto es, la inferencia o mejor dicho, la petición de acción que produce el enunciado: son peticiones de acción expresadas en presente de imperativo ('tráeme un café', 'estudia más', 'acompáñame al cine'). Este problema, ya lo subraya la NGLE 
(2009: §23.15ñ) diciendo que "La alternancia del condicional con el presente no siempre se obtiene, en cambio, cuando se usa el primero para expresar un deseo que se considera actual. (...) Me gustaría decir algo no significa me gusta decir algo" y Haillet (2002: 70) para el francés:

'Cela me ferait plaisir de monter sur scène à tes côtés' ne commute pas avec 'cela me fait plaisir de monter sur scène à tes côtés', d'où l'impossibilité de considérer l'énoncé au conditionnel comme une version atténuée de l'assertion au présent. Il s'agit d'un conditionnel d'hypothèse ne produisant pas d'effet de désactualisation.

Ahora bien, ninguno de los dos da explicaciones. Intentaremos demostrar por qué no se da esta equivalencia. Formulamos la hipótesis de que es modal porque es una forma derivada del condicional "no factual": por una parte, se puede sobreentender una prótasis y de ese modo, trasladar la situación a un mundo posible. Por otra, hace falta sacar una implicatura para interpretarlo de modo adecuado, de suerte que coexisten dos lecturas: una lectura semántica o literal que sitúa la acción en un mundo posible, y una lectura pragmática o inferencial en la que se lee el enunciado a partir del presente. En primer lugar, se puede sobreentender una prótasis: en (27) y (28) se recupera mediante el contexto y en (29) y (30), ya está contenida la prótasis en la misma frase, como se comprobará en lo ejemplos (31) a (34).

(31) De buena gana me tomaría un café (si pudiese).

(32) ¿De qué color es este jersey? -Yo diría que es azul (si me lo preguntaras).

(33) Si me acompañaras al cine, me encantaría.

(34) Si me acompañaras al cine, me gustaría.

En segundo lugar, distinguiremos entre lectura literal y lectura inferencial. En (31) puede afirmarse que se utiliza un condicional no factual ya que es posible, mediante el contexto, recuperar una prótasis y obtener 'si pudiese, me tomaría un café'. Se sitúa 
la acción de 'tomarme un café' en otro mundo posible, distinto del de mi presente: $\langle$ si $p, q\rangle$, donde se sobreentiende $q$. Es una construcción condicional de tipo irreal porque implica una negación de presente ('no me tomo un café ahora mismo') ${ }^{17}$. La segunda lectura de tipo inferencial es que -en un determinado contexto- deseo beber un café, y le pido a mi interlocutor que me traiga uno. Los casos (33) y (34) plantean problemas porque como se ha dicho, no equivalen a un presente. En el caso de (33), estamos diciendo que si en otro mundo, me acompañaras al cine, me encantaría. Se desencadena la implicatura: deseo que me acompañes al cine y acompáñame al cine ahora mismo. El caso de (34) plantea cuestiones interesantes. En principio este ejemplo debería entenderse como en otro mundo, si me acompañaras al cine, me gustaría. Sin embargo, en muy pocos casos se le da este sentido. No significa en otro mundo, me daría gusto o me agradaría, sino en el mundo actual, deseo; creemos que la segunda lectura-en principio, inferencial ya que expresa el deseo-es la única posible, al menos en este contexto.Comparemos el español con el francés. En español, existen las dos lecturas: "Me gustaría que me acompañaras al cine" significa en el mundo actual deseo o bien "si me acompañaras al cine, me gustaría", significa en otro mundo posible, si me acompañaras al cine, me agradaría y es un condicional no factual (sin petición de acción). En cambio, en francés existe una sola lectura: "j'aimerais"solo quiere decir deseo en el mundo actual y nunca me daría gusto en otro mundo. Esto se comprobará con el ejemplo

(35) J'aimerais que tu m'accompagnes au cinéma

que tan solo cobra el sentido de quiero ahora que me acompa$\tilde{n} e s .{ }^{18}$ La equivalencia con la construcción condicional no se da ya que resultaría inaceptable la frase (36).

17 Ver al respecto discusión de Veiga (1991: 155).

18 Para Veiga (1991) son “irreales", para Rivero (1978) "potenciales".En nuestra opinión, pragmáticamente son potenciales: puede realizarse la acción de 'ir al cine'. Semánticamente son irreales: hago como si no me gustara ahora mismo ("falsas irreales" según Veiga 1991: 155). 
(36) *Si tu m’accompagnais au cinéma, j'aimerais (cela) ${ }^{19}$.

J'aimerais se ha gramaticalizado por completo y tan solo significa deseo en el presente -nunca j'aimerais en otro mundo-, mientras que me gustaría aún puede usarse en los dos contextos. Para resumir, es modal el condicional de cortesía o de atenuación porque se asemeja a un condicional no factual, ya que gracias al contexto, se puede recuperar una prótasis -ría permite situar la acción en un mundo posible $(\langle$ si $p, q\rangle)-\mathrm{y}$ de esta construcción condicional, se hace una interpretación anclada en el presente de enunciación: se saca una implicatura de presente que permite reinterpretar el enunciado como una petición de acción o como una simple atenuación de lo dicho. Nos centraremos ahora en el condicional de conjetura y en el condicional del rumor.

\section{El condicional de conjetura y el condicional del rumor ("pe- riodístico").}

\subsection{El condicional de conjetura}

El condicional de conjetura es un condicional de probabilidad o epistémico que equivale a "probablemente + imperfecto" (NGLE 2009: §23.15j) como se observará con el ejemplo (37):

(37) Juan tendría entonces unos 40 años.

equivale a 'Juan entonces tenía probablemente unos 40 años'. En algunos casos, llega a ser equivalente de un pretérito simple:

(38) Pedro llegaría entonces tarde.

equivale a 'Pedro entonces llegó probablemente tarde'. ${ }^{20}$

19 De hecho, sería aceptable la frase: Su tu m'accompagnais au cinéma, cela me plairait. Concluimos que la prótasis puede ser retomada como sujeto (cela me plairait) y no como complemento (j'aimerais cela).

20 Agradezco este comentario a la profesora Cristina Sánchez. 
¿Cómo puede explicarse el hecho de que sea modal? Intentemos ver si se puede interpretar como un condicional no factual. Cuando decimos Tendría entonces 40 años, la situación de tener entonces 40 años no se sitúa en un mundo posible de igual forma que tendría 40 años, si no hubiera muerto, enunciado no factual contrafáctico (condición sin hipótesis), cuya apódosis depende de una prótasis, y que significa o implica en el presente no tiene 40 años. $\mathrm{Al}$ contrario, en tendría entonces 40 años se expresa una hipótesis sin condición: No sabemos si tenía o no tenía 40 años entonces y a la vez es probable que tuviera 40 años. Para explicar dicho valor, Rojo \& Veiga (1999) y Veiga (1991: 45) recurren a la dislocación de vectores: "al sustituirse la orientación de posterioridad por una de simultaneidad, la forma pasa a expresar un contenido modal de probabilidad". Ahora bien, si expresa simultaneidad con un momento pasado, lo que obtenemos es un pretérito imperfecto: tenía 40 años. ¿Cómo llega a producir probabilidad la dislocación de vectores?

Podemos formular la hipótesis de que el condicional (terminación en -ría) funciona como un operador epistémico de posibilidad, pero cabe notar como dice MacFarlane (2011: 144) que "[E]pistemic [modals] have something to do with knowledge. But knowledge presupposes a knower or knowers". El hablante expresa su punto de vista, el de considerar como hipotética (posible) una situación pasada: no sabe si ha ocurrido o no. La hipótesis que formulamos es que -ría funciona como un operador epistémico de posibilidad que opera sobre un enunciado en pretérito imperfecto o en pretérito simple de modo que tendríamos:

[es posible [pretérito imperfecto / pretérito simple]]

\subsection{El condicional del rumor}

El condicional del rumor o periodístico quizás sea el menos ortodoxo de todos los condicionales dentro de la gramática del español. Si bien existe una amplia bibliografía sobre el tema en francés y se le ha dado varios nombres, journalistique (Gosselin 2001), de ci- 
tation (Kozen \& Nølke 1990), d'altérité énonciative (Haillet 1995), de non prise en charge (Abouda 2001), no se le ha prestado tanta atención en español. Se le llama condicional de información no asegurada (Lapesa 1977), periodístico (Veiga 1991), del rumor (Casado Velarde 1995; NGLE 2009). Hasta fechas recientes, se condenaba el uso de esta forma. Gili Gaya (1943: §129, nota 6) decía lo siguiente:

En el siglo actual, aparecen con alguna frecuencia en los periódicos de todos los países hispánicos noticias redactadas de este modo [...]. Evidentemente se trata de telegramas traducidos del inglés o del francés. [...] Hay que consignar sin embargo, que se trata de un uso reciente y muy limitado.

El Libro de Estilo(El País, 1991: 124) más tarde prohibiría su uso:

El uso del condicional en ese tipo de frases queda terminantemente prohibido en el periódico. Además de incorrecto gramaticalmente, resta credibilidad a la información.

Lázaro Carreter (2001: 386) habla de 'jerga informativa' explicando que:

Es puro francés. $X$ rencontrerait $Y$ prochainement. [...] Pero el idioma cuenta, para advertir que algo no está comprobado, con propios y acreditados recursos: se dice..., Parece ser que..., Aseguran..., Es probable o posible que...

En fechas más recientes, Grijelmo, (29/07/2012 El País, "el rumor enmascarado") lo describe de la forma siguiente:

Y esos "habría" "estaría" o "sería" no hacen sino narrar algo que el periodista no puede dar por seguro. Es decir, algo que no se ha comprobado o que no se puede atribuir a una fuente que lo asuma como cierto. Un rumor. 
Para terminar con esta presentación, añadiremos que la NGLE (2009 § 23.15m) no condena su uso, definiéndolo como "la variante del condicional de conjetura que se usa a menudo para presentar las informaciones de forma cautelosa".Todos los estudiosos (Lapesa 1977; Veiga 1991; Casado Velarde 1995, NGLE 2009) están de acuerdo en que un condicional del rumor permite presentar un hecho sin que el hablante se comprometa con la verdad de lo aseverado. Por otra parte, adquiere un valor evidencial que deja ver que la fuente de la información no es el propio hablante. Ahora bien, si el condicional del rumor es "una variante del condicional de conjetura", ¿en qué se parecen y en qué difieren? Los enunciados (39), (40) y (41) son claros ejemplos del condicional del rumor (para una definición, véanse Veiga 1991; Casado Velarde 1995; Vatrican 2010).

(39) Según otras informaciones, los ataques habrían durado toda la noche. (Fernández López 2007).

(40) Todos los españoles estarían a salvo, menos uno, el mallorquín Guillermo Gual de 68 ("Abandonados y enfadados", El País, 15/01/12).

(41) Vicky Xipolitakis viajaría a Dubai para ver a Maradona. La vedette partiría rumbo al emirato para "consolar" al recientemente separado "Diez". (La Gaceta, 8/03/14, Argentina).

Se parecen el condicional del rumor y el de conjetura en que ambos presentan, a grandes rasgos, un hecho como hipotético, parafraseable por puede que ${ }^{21}$, de modo que la equivalencia entre (a) y (b) se da tanto para el condicional del rumor en (42), (43) y (44) como para el condicional de conjetura en (45):

(42) a. Según otras informaciones, los ataques habrían duradotoda la noche. (Fernández López 2007).

b. Puede que los ataques hayan durado toda la noche.

21 Lo que nos interesa aquí es comprobar que tanto el condicional del rumor como el de conjetura comparten el rasgo de expresar una hipótesis equivalente a puede que. 
(43) a. Todos los españoles estarían a salvo, menos uno, el mallorquín Guillermo Gual de 68 ("Abandonados y enfadados", El País, 15/01/12).

b. Puede que todos los españoles estén a salvo.

(44) a. Vicky Xipolitakis viajaría a Dubai para ver a Maradona. La vedette partiría rumbo al emirato para "consolar" al recientemente separado "Diez". (La Gaceta, 8 de marzo de 2014, Argentina).

b. Puede que Vicky Xipolitakis vaya a viajar a Dubai.

(45) a. Juan tendría entonces unos 40 años.

b. Puede que Juan tuviera 40 años.

Ambos expresan la modalidad epistémica, esto es, proposicional, de hipótesis. Sin embargo difieren en dos cosas: en que tienen enfoques temporales distintos y en que el condicional del rumor es evidencial mientras que el de conjetura no lo es. Primero, difieren en el enfoque temporal. Como se observará a continuación, mientras que el condicional del rumor tiene enfoque de pretérito compuesto en (46), de presente en (47) o de futuro en (48), el condicional de conjetura solo tiene enfoque temporal de pretérito imperfecto en (49) o de pretérito simple en (50)

(46) a. Según otras informaciones, los ataques habrían durado toda la noche.

b.Puede que [los ataques han durado toda la noche] = pretérito compuesto

(47) a. Todos los españoles estarían a salvo, menos uno, el mallorquín Guillermo Gual de 68.

b. Puede que [todos los españoles estén a salvo] = presente

(48) a. Vicky Xipolitakis viajaría a Dubai para ver a Maradona. La vedette partiría rumbo al emirato para "consolar" al recientemente separado "Diez". (La Gaceta, 8 de marzo de 2014, Argentina) 
$=$ futuro

b. Puede que [V. Xipolitakis viajarálva a viajar a Dubai.]

(49) a. Juan tendría entonces unos 40 años. imperfecto

b.Puede que [Juan entonces tenía unos 40 años] = pretérito

(50) a. Ayer Pedro llegaría tarde.

b. Puede que [ayer Pedro llegó tarde] = pretérito simple

Como acaba de verse, la morfología de condicional -ría opera sobre una situación presente o futura mientras que en el caso del condicional de conjetura, opera sobre una situación que se da en pretérito imperfecto o pretérito simple. Se observará que un mismo verbo (tendría) combinado con un adverbio temporal con valor prospectivo (próximamente), produce un condicional del rumor como en (51), mientras que combinado con un adverbio temporal de pasado (entonces), produce el condicional de conjetura como en (52):

(51) Sevilla tendría próximamente carrera de psicología para los jóvenes que deseen estudiar esta carrera en el municipio. (Canal Vivavisión, 08/07/2014)

(52) Juan tendría entonces unos 40 años.

En segundo lugar, difieren en que solo el condicional del rumor tiene componente evidencial. Recordemos que la evidencialidad es la fuente de la información (Aikhenvald 2004), la cual puede ser directa-visual, auditiva- o indirecta -reportativa o inferencial-.El condicional es evidencial porque da a entender que el hablante cita, esto es, reproduce lo que alguien le ha dicho, sin asegurar que sea verdad. Forma parte de la evidencialidad de tipo 'reportativo' ('hearsay' in Anderson 1986: 289). Obsérvense los siguientes ejemplos.

(53) a. Según otras informaciones, los ataques habrían durado toda la noche. (Fernández López 2007) 
b. $\{$ Dicen que $\}$ los ataques $\{$ supuestamente $\}$ han durado toda la noche.

c. Puede que sea verdad que los ataques hayan durado toda la noche.

(54) a. Todos los españoles estarían a salvo, menos uno, el mallorquín Guillermo Gual de 68. salvo.

b. $\{$ Dicen que $\}$ todos los españoles $\{$ supuestamente\} están a

c. Puede que sea verdad que todos los españoles estén a salvo.

(55) a. Vicky Xipolitakis viajaría a Dubai para ver a Maradona. La vedette partiría rumbo al emirato para "consolar" al recientemente separado "Diez".

b. $\{$ Dicen que $\}$ V. X. va a viajar\{supuestamente $\}$ a Dubai.

c. Puede que sea verdad queV. X.vaya a viajar a Dubai.

(56) a. Juan tendría entonces unos 40 años.

\# b. \{Dicen que\} Juan tenía \{supuestamente\} 40 años.

\# c. Puede que sea verdad que Juan tuviera unos 40 años.

Se da la equivalencia entre (a), (b) y (c) en los tres casos de condicional del rumor (53), (54) y (55), donde el morfema -ría equivale a dicen que o supuestamente (otro hablante), y también a puede que sea verdad; en cambio, estas equivalencias no se dan con el condicional del rumor: de ninguna forma (56 a) equivale a (56 b) o a (56 c).

Cabe apuntar dos cosas. La primera es la cuestión tan debatida de la relación entre evidencialidad y modalidad epistémica. Existen dos teorías: algunos como Matthewson et al. (2007) o McCready \& Ogata (2007) consideran que es una subclase de la modalidad epistémica y por tanto, según ellos, algunos modales epistémicos son evidenciales. Otros como Aikhenvald (2004) consideran que la evidencialidad y la modalidad epistémica son 
dos clases distintas. La segunda cuestión es que aparece cierta paradoja a la hora de hablar de condicional evidencial, puesto que, en principio, la evidencialidad solo indica la fuente de una información, esto es, la fuente de un hecho seguro; como dice Anderson (1986: 273), “Evidentials express the kinds of evidence a person has for making factual claims". Con el condicional no hay ningún hecho seguro, y por tanto ninguna información, o al menos, ninguna información segura.

Para terminar, ¿qué relación mantienen la modalidad epistémica con la evidencialidad en el caso del condicional? A esta pregunta, podemos contestar de dos formas. Puede decirse que el condicional evidencial solo significa "me han contado que" ("dicen que"), y a consecuencia de ello, el sentido de posibilidad se infiere del evidencial: la posibilidad no está a cargo del hablante. O bien la posibilidad está a cargo del hablante: el hablante no sabe si lo que le han dicho es verdad o no (no exactamente si el propio hecho ha ocurrido o no), de modo que podemos hacer la siguiente pregunta: ¿La posibilidad de que sea verdad o no cabe en la semántica del morfema -ría o es una inferencia? Propondremos a modo de hipótesis que -ría funciona como un operador epistémico que no opera sobre el enunciado -como en el caso del condicional de conjetura- sino sobre la enunciación, sobre la fuerza ilocutiva de lo aseverado; de modo que tendríamos el esquema siguiente:

[[[Es posible [ser verdad [futuro / presente / pretérito compuesto]]]

\section{Conclusión}

Nuestro objetivo era explicar en qué medida puede afirmarse que estos cuatro tipos de condicionales, no factual, de cortesía, de conjetura y del rumor, son modales y analizar cómo funciona la morfología de condicional -ría en los cuatro casos. Hemos visto en la primera parte que el condicional se ha creado a partir de una perífrasis que en su origen tenía valor modal de obligación, de necesidad epistémica en la medida en que se aplicaba a una proposición. 
A continuación, se ha expuesto que la doble naturaleza del condicional, temporal y modal, ha sido objeto de debates a lo largo de los siglos. Si bien se ha podido considerar en la historia como un modo, hoy en día se suele aceptar la idea de que es un tiempo con valores modales. Hemos analizado cada uno de ellos y llegado a la conclusión de que se podían agrupar en dos clases: en el primer grupo, que abarca el condicional no factual y el de cortesía, el morfema -ría tiene por función trasladar la acción a un mundo posible mediante una condición $(<$ si $p, q>)$. El condicional no factual aparece en una construcción condicional con o sin prótasis explícita y el enunciado se lee a partir del mundo posible. El condicional de cortesía/ atenuación aparece en una construcción condicional del tipo $<$ si $p$, $q>$ y el morfema -ría traslada la acción a un mundo posible pero se lee el enunciado a partir de una implicatura de presente (deseo, petición de acción, aceptación de lo dicho). En el segundo grupo que abarca el condicional de conjetura y el del rumor, el morfema -ría puede considerarse como un operador modal epistémico de posibilidad. Con el condicional de conjetura el morfema -ría opera sobre el enunciado, el cual tiene enfoque de pretérito imperfecto o de pretérito simple, de modo que tendríamos el siguiente esquema:

[Operador modal epistémico [(PRET. IMPER) / (PRET. SIMPLE)]]

Con el condicional del rumor, el morfema -ría hace también de operador epistémico de posibilidad pero en este caso, opera sobre la enunciación, esto es, sobre la fuerza ilocutiva de lo aseverado; el enfoque temporal del enunciado debe ser de presente, de futuro o de pretérito compuesto, de modo que el esquema que proponemos es el siguiente:

[Operador modal epistémico [ser verdad [(PRES) / (FUT) / (PRET.COMP)]]]. 


\section{Biliografía}

Aвоuda, L., "Les emplois journalistique, polémique et atténuatif du conditionnel. Un traitement unitaire", in P. Dendale \& L. Tasmowski (eds.), Le conditionnel en français, Paris, Klincksieck, 2001, pp. 277-294.

Aikhenvald, A., Evidentiality, Oxford, Oxford University Press, 2004.

Albelda, M.\& Briz, A., "Aspectos pragmáticos. Cortesía y atenuantes verbales en las dos orillas a través de muestras orales", en Aleza M. y Enguita, J. M,La lengua española en América. Normas y usos actuales, Universitat de València, 2010, pp. 237-260. (Consultable en línea: http://www.uv.es/aleza/.)

Anderson, "Evidentials, Paths of change, and mental maps: typologically regular asymmetries", W. Chafe \&J. Nichols (eds),Evidentiality, Norwood, Ablex, 1986, pp. 273-312.

Bello, A., Gramática de la lengua castellana, Madrid, Edaf, 1984 [1847].

BRIZ, A., "Sobre el fenómeno dela cortesía", en Pragmática sociocultural: estudios sobre el discurso de cortesía en español, D. Bravo \& A. Briz (eds), 2004, pp. 67-93.

Carrasco Gutiérrez, A., "El tiempo verbal y la sintaxis oracional. La consecutio temporum", en Bosque, I. y Demonte, V. (eds.), Gramática descriptiva de la lengua española, 1999, pp. 3061-3130.

Casado Velarde, M., "El lenguaje de los medios de comunicación", in M. Seco \& G. Salvador (eds.), La lengua española hoy, Madrid, Fundación Juan March, 1995,pp. 153-164.

Castillo Lluch, M., “Distribución de las formas analíticas y sintéticas de futuro y condicional en español medieval", in María Teresa Echenique Elizondo/Juan Sánchez Méndez (eds.) : Actas del Quinto Congreso Internacional de Historia de la Lengua Española, Madrid : Gredos, 2002, I, p. 541-549. 
Company Company, C., “Tiempos de formación romance II. Los futuros y condicionales", C. Company Company (coord.), Sintaxis histórica de la lenguaespañola. Primera parte: la frase verbal. México: Fondo de Cultura Económica-UNAM, 2006,pp. 349-423.

Company Company, C., "Los futuros en el español medieval: sus orígenes y su evolución", NRFH, XXXIV, 1985-1986, pp. 49107.

Company, C. \& Medina, A., "Sintaxis motivada pragmáticamente: futuros analíticos y futuros sintéticos en el español medieval", Revista de Filología española, Tomo 79, Fasc. 1-2, 1999, pp. 65-100.

Dendale, P.,\& L. Tasmowski, Le conditionnel en français, Paris, Klincksieck, 2001, pp. 7-18.

El PAís, "El condicional de rumor", in Libro de estilo, Madrid, Ed. El País, 1991.

EsCANDELl, M.-V, 1995, “Cortesía, fórmulas convencionales y estrategias indirectas", Revista Española de Lingüística, 25, 1, pp. 31-66.

Escandell, M.-V, Fundamentos de semántica composicional, Barcelona, Ariel, 2004.

Fernández López, J., “Condicional de rumor o de información no asegurada", 2007, www. culturitalia.uibk.ac.at/hispanoteca.

Frege, G., 1971, Ecrits logiques et philosophiques, Paris, Points Seuil.

Gili Gaya, Curso superior de sintaxis española, Barcelona, Vox, 1948[1993].

Girón Alconchel, J.L., “La morfología de los futuros: de Nebrija a la Academia (1771)", Anuario de lingüística hispánica, Vol. 12-13, 1, 1996-1997, pp. 55-72.

Gosselin, L., "Relations temporelles et modales dans le 'conditionnel journalistique'”, in P. Dendale \& L. Tasmowski (eds.), Le conditionnel en français, Paris, Klincksieck, 2001, pp. 45-66. 
Grice, P., Studies in the Way of Words, Cambridge, Harvard University Press, 1989.

Grice, P., "Logic and conversation", en Syntax and Semantics III: Speech Acts, P. Cole \& J. L. Morgan (eds), Nueva York, Academic Press, 1975, pp. 41-58.

Grijelmo, Á., “El rumor enmascarado”, El País, 29/07/2012. Disponible en línea: http://elpais.com/elpais/2012/07/19/ opinion/1342716341_843818.html.

HaIllet, P.,Le conditionnel en français. Une approche polyphonique, Paris, Ophrys, 2002.

Haverkate, H., La cortesía verbal. Estudio pragmalingüístico, Madrid, Gredos, 1994.

IATRIDOu, S., "The Grammatical Ingredients in Counterfactuality", Linguistic Inquiry 31, 2, 2000, pp. 231-270.

Kearns, K., Semantics, New York, Palgrave Macmillan, 2011.

Kerbrat-Orecchioni, C., “¿Es universal lacortesía?”, en Pragmática sociocultural: estudios sobre el discurso de cortesía en español, D. Bravo \& A. Briz (eds), 2004, pp. 39-65.

Korzen, H. \& Nølke, H., "Projet pour une théorie sur les emplois du conditionnel", Actes du XIème Congrès des RomanistesScandinaves, Trondheim, Université de Trondheim, 1990, pp. 301312.

Kratzer, A. 1991: «Modality », in A. von Stechow y D. Wunderlich (eds.), Semantik, Berlín-Nueva York, De Gruyter, pp. 639-650.

Kronning, H., “Le conditionnel 'journalistique' : médiation et modalisation épistémiques", XVème congrès de romanistes scandinaves, 12-17 agosto, 2002, pp. 561-575.

LACA, B., “Tiempo, aspecto y la interpretación de los verbos modales en español", Lingüistica ALFAL 17, 2005, pp. 9-44.Disponibleenlínea:http://halshs.archives-ouvertes.fr/docs/00/10/46/39/ PDF/mod.aspALFAL2.pdf 
LAPESA, R., "Tendencias y problemas actuales de la lingüística española", in R. Lapesa (ed.), Comunicación y lenguaje, Madrid, Karpos, 1977, pp. 203-229.

Lázaro Carreter, F.,El dardo en la palabra, Barcelona, Galaxia Gutenberg/ Círculo de lectores, 2001.

LLOYD, P.M., Del latín al español: fonología y morfología históricas de la lengua española, Madrid, Gredos, 1993.

Lyons, J., Sémantique linguistique, Paris, Larousse, 1990.

Macfarlane, J., "Epistemic Modals are Assessment-Sensitive", in Epistemic Modality, A. egan \& B. Weatherson (eds), Oxford, Oxford University Press, 2011, pp. 144-178.

Mccready, E. \& Ogata, N., “Evidentiality, Modality and Probability",Linguistics and Philosophy, 30 (2), 2007, pp. 147-206.

Matthewson, L., Davis, H. \& Rullman, H., "Evidentials as epistemic modals. Evidence from St'át'imcets", Linguistics Variation Yearbook, John Benjamins Publishing Company, vol. 7, 1, 2007, pp. 201-254.

Merle, J.M., Jean-Marie Merle, Étude du conditionnel français et de ses traductions en anglais, Paris / Gap, Ophrys, 2001, pp. 2429.

Molina Redondo, J.A., Gramática avanzada para la enseñanza del español, P. Barros García y E. Morales Manrique (ed), Universidad de Granada, 2011

Montolío, E., "Las construcciones condicionales", en Bosque, I. y Demonte, V. (eds.), Gramática descriptiva de la lengua española, 1999, pp. 3644-3737.

Moss, S., "On the pragmatics of counterfactuals", Nô̂s, 46, 3 , 2012, pp. 561-586.

Penny, R., Gramática histórica del español, Barcelona, Ariel, 2001. 
Pato, E., La sustitución del imperfecto de subjuntivo por el condicional simple y el imperfecto de indicativo en el castellano septentrional peninsular. (Estudio de variación dialectal), UniversidadAutónoma de Madrid, tesis doctoral, 2003.

Quine, W V., Methods of Logic, New York: Holt, Rinehart and Winston,1959.

Real Academia Española- Asale, Nueva Gramática de la Lengua Española, Madrid, Espasa, 2009.

RidRuejo, E., Las estructuras gramaticales desde el punto de vista histórico, Madrid, Síntesis, 1989.

Rivero, M. L., “Un ejemplo de metodología de filosofía analítica en la semántica lingüística: la cortesía y los actos verbales", Revista española de lingüística, vol. 8, núm. 1, 1978, pp. 77-103.

Rojo, G. \& Veiga, A., "El tiempo verbal. Los tiempos simples”, en Bosque, I. y Demonte, V. (eds.), Gramática descriptiva de la lengua española, 1999, pp. 2867-2934.

Searle, J., Speech acts, Nueva York, Cambridge University Press, 1969.

Stalnaker, R., "A theory of Conditionals", in N. Rescher (ed), 1968, Studies in Logical Theory, 98-112.

"Conditional Propositions and Conditional Assertions", in Epistemic Modality, A. Egan \& B. Weatherson (eds), Oxford, Oxford University Press, 2011, pp. 227-248.

VAtrican, A., “La modalité et le conditionnel de rumeur en espagnol et en français", Modèles linguistiques, tome XXXI, vol. 62, 2010, pp. 83-94.

(Disponible en línea: http://hal.archives-ouvertes.fr/hal00675063/)

VAtrican, A.,"El condicional de cortesía en español: la hipótesis como forma de atenuación", in A. Cabedo Nebot, M. J. Aguilar Ruiz \& E. López-Navarra Vidal (eds), Estudios de lingüística: 
investigaciones, propuestas y aplicaciones, Universitat de València, 2013, pp. 469-480.

Veiga, A.,Condicionales, concesivas y modo verbal en español (Verba Anexo 34), Santiago de Compostela, Universidad de Santiago de Compostela, 1991.

Von Fintel, K., "Counterfactuals in a Dynamic Context." In Ken Hale: A Life in Language, Michael Kenstowicz (ed), MIT Press, Cambridge, 2001.

Axelle Vatrican

Universidad de Toulon-Babel EA2649 /

Universidad COMPLUTENSE DE MADRID 
